\title{
Real World Patient Perceptions of the use of Tazarotene $0.1 \%$ Foam in the Treatment of Acne Vulgaris
}

James Q. Del Rosso DO, FAOCD, FAAD; ${ }^{1}$ Corey L. Hartman MD FAAD; ${ }^{2}$ Caitlin Lewis PhD; ${ }^{3}$ Rhonda Schreiber MSRN. ${ }^{4}$

\section{INTRODUCTION}

(AV) is the most common inflammatory skin disorder seen in outpatient dermatology clinics in the United States. Both adolescents and adults of all races and there are several adverse psychosocial consequences that impair quality of life. Continued patient compliance with topical therapies is a recognized barrier to optimal tormulation strongly influences such as AV. ${ }^{1}$ Patient satisfaction with a topical vehicle the only retinoid approved for use in a foam vehicle and is well established as an studies evaluating tazarotene $0.1 \%$ foam for AV supported positive patient experiences Wh both therapeutic outcomes and formulation characteristics. ${ }^{\circ}$ These overall positive tient experiences from clinical trial $0.1 \%$ foam to gather perspectives on its use in "real world" clinical practice. Patients with on the face and/or trunk who were being treated with tazarotene $0.1 \%$ foam were

\section{METHODS}

Around 300 survey kits were distributed across the USA to capture data from diverse geographical areas and climates

and in to capture use in the winter on well as non-winter months

Feedback was gathered on overall patient satisfaction with use of the product, percen therapeutic impact on $\mathrm{AV}$, and topical vehicle preference

Ater registering at baseline patients completed surveys within 3 days of the 2, 4, instency of reporting and objectivity of analysis

- A total of 372 patients participated in the surveys through week 12 with a broad A $n$ values $=$ number of respondents to each question in results graphs)

\begin{tabular}{|ccc|}
\hline Total Respondents Through Week $\mathbf{1 2} \mathbf{n}=\mathbf{3 7 2}$ & $\mathbf{n}(\%)$ \\
\hline \multirow{3}{*}{ Gender } & Male & $118(31.7)$ \\
& Female & $252(67.7)$ \\
& Other & $2(0.6)$ \\
\hline \multirow{3}{*}{ Age } & $12-20$ & $107(29)$ \\
& $21-30$ & $121(33)$ \\
& $31+$ & $143(38)$ \\
\hline \multirow{4}{*}{ Race } & Caucasian & $252(68)$ \\
& African American & $36(10)$ \\
& Hispanic or Latino & $46(12)$ \\
& Other & $37(10)$ \\
\hline
\end{tabular}

\section{DISCUSSION}

drug delivery and therefore impact safety, efficacy and tolerability. ${ }^{3}$ In recent years aqueous-based foam formulations have become a preferred vehicle in treating skin disease as their favorable likelihood that adherence to treatment regimens including these foam vehicles will also improve. However, early foam formulations were positioned for use in diseases that affected large body surface areas, leading to a general belief amongst clinicians that the foams were only suitable for large areas, such as the trunk, due to their spreadability. ${ }^{3}$ In commonly associated with early formulations, and lack of familiarity with proper application techniques has also led clinicians to avoid use of tazarotene $0.1 \%$ foam on the face and during the dry winter months, regardless of its novel foam formulation and the efficacy shown in Phase III trials. The results of specific questions from the surveys that address these historical perceptions of topical tazarot
tabulated and can be seen in the graphs to the right.

\section{CONCLUSION}

The data presented here, captured from patients who had completed 12 weeks of significant sample size with diversity across gender, race, and age. These results contradict many prior assertions regarding topical tazarotene products. Patient satisfaction levels high across gender, age and race, regardless of the time of year the treatment was used or the area of the body being treated, and were higher with consistent use vs. inconsisten use. Overall, these real-world responses support the results of patient questionnaires from patients as an effective, tolerable, and easy-to-use treatment option for $\mathrm{AV}$ of the face and

\section{REFERENCES}

\section{RESULTS}

\section{Overall Satisfaction Week 12 ( $n=371^{*}$ )}
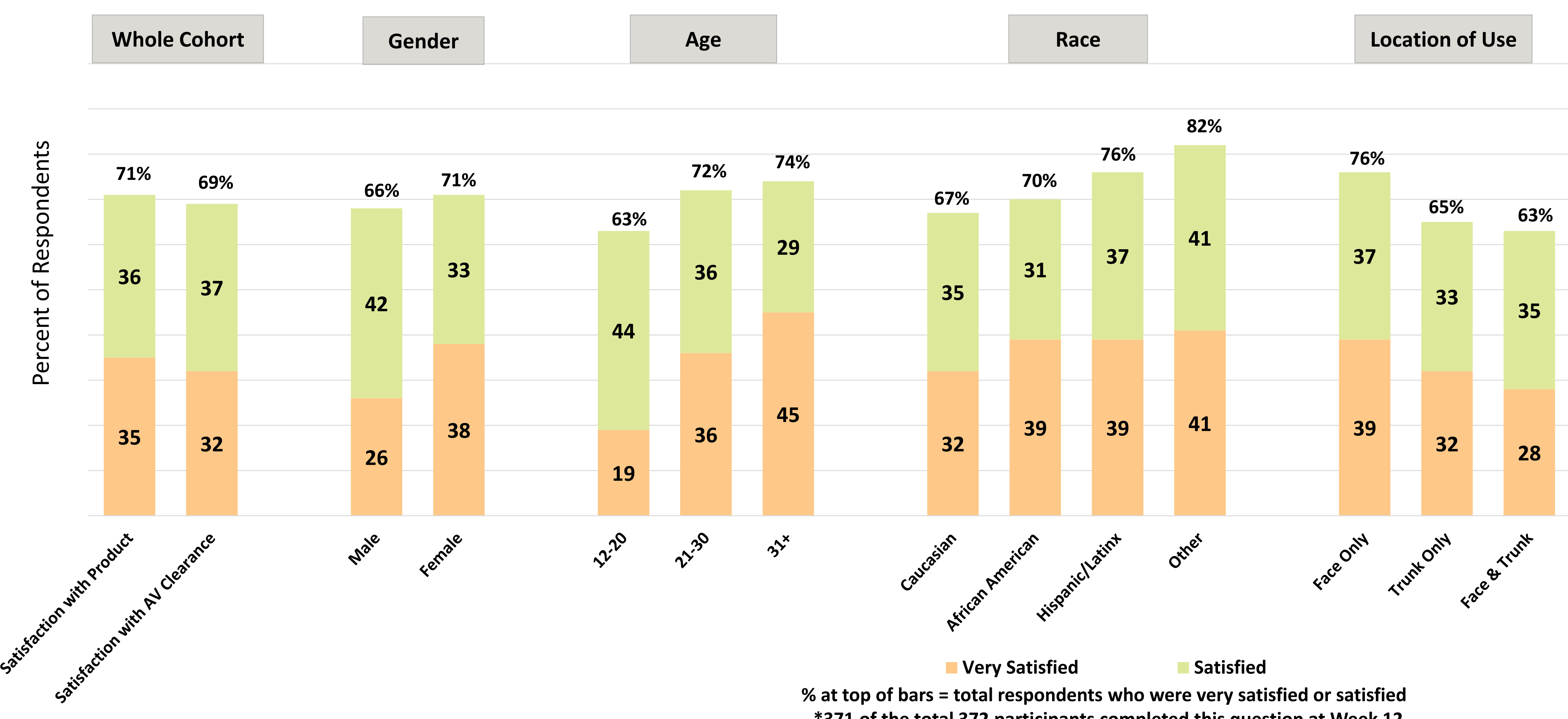

Season of Use

Consistency of Use question at Week $12,71 \%$ stated they were either very satisfied or satisfied with tazarotene $0.1 \%$ foam and $69 \%$ were satisfied with the clearance of acne achieved during the survey period. While satisfaction was avrable overall the highest levels were reported by the following subsets: female patients, those who used the product on their face only, those who used the product in winter, and those who used the product While non-white responders reported slightly higher levels of satisfaction, the differences between the sub-groups are also low. While common perception is that foams are suited better to large treatment areas and topical retinoids are poorly tolerated on the face, $77 \%$ of participants in these surveys were using tazarotene $0.1 \%$ foam on the face and the data showed higher satisfaction levels in those using the product on the face only vs. those using it on the trunk only or face and trunk. When satisfaction was rated based on season of use, tazarotene $0.1 \%$ foam again showed results that contradict traditional views that satisfaction slightly higher. Participants who reported using the product daily or every other day on every survey were defined as 'Consistent Use' ( $\mathrm{n}=215)$. Those who marked an option other than daily or every other day use on any survey were defined as 'Some Inconsistent Use' ( $n=156)$. A subset of the latter group were those who never reported daily or every other day use, defined as 'Only Inconsistent Use' ( $n=61)$. treatment outcomes.

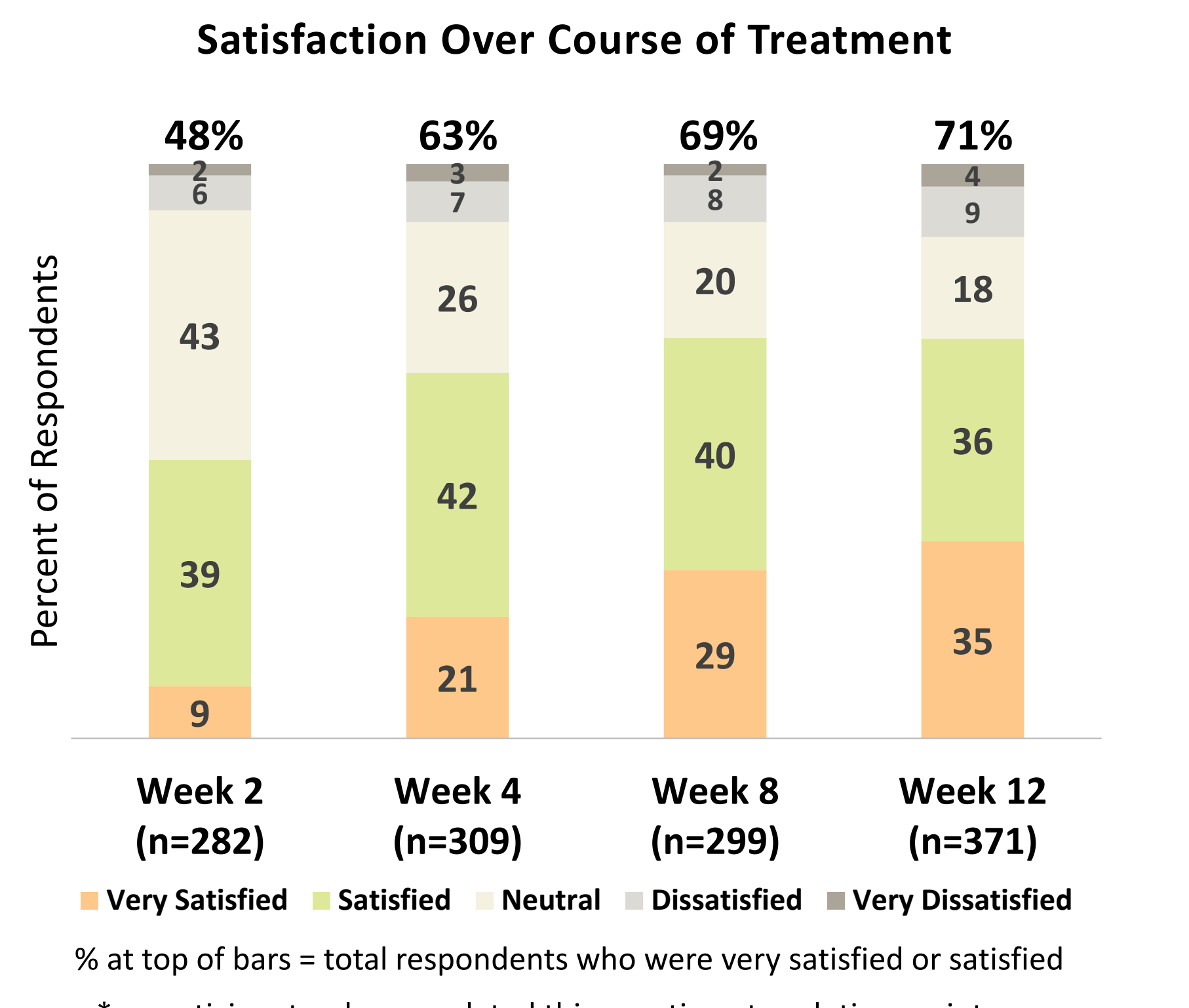

\section{(a)} $0.1 \%$ foam on all surveys. Patient satisfaction with the product remained very low throughout. At week $12,71 \%$ of respondents indicated they were very satisfied or satisfied with tazarotene $0.1 \%$ foam and $67 \%$ indicating that they were likely or very likely to continue use of the
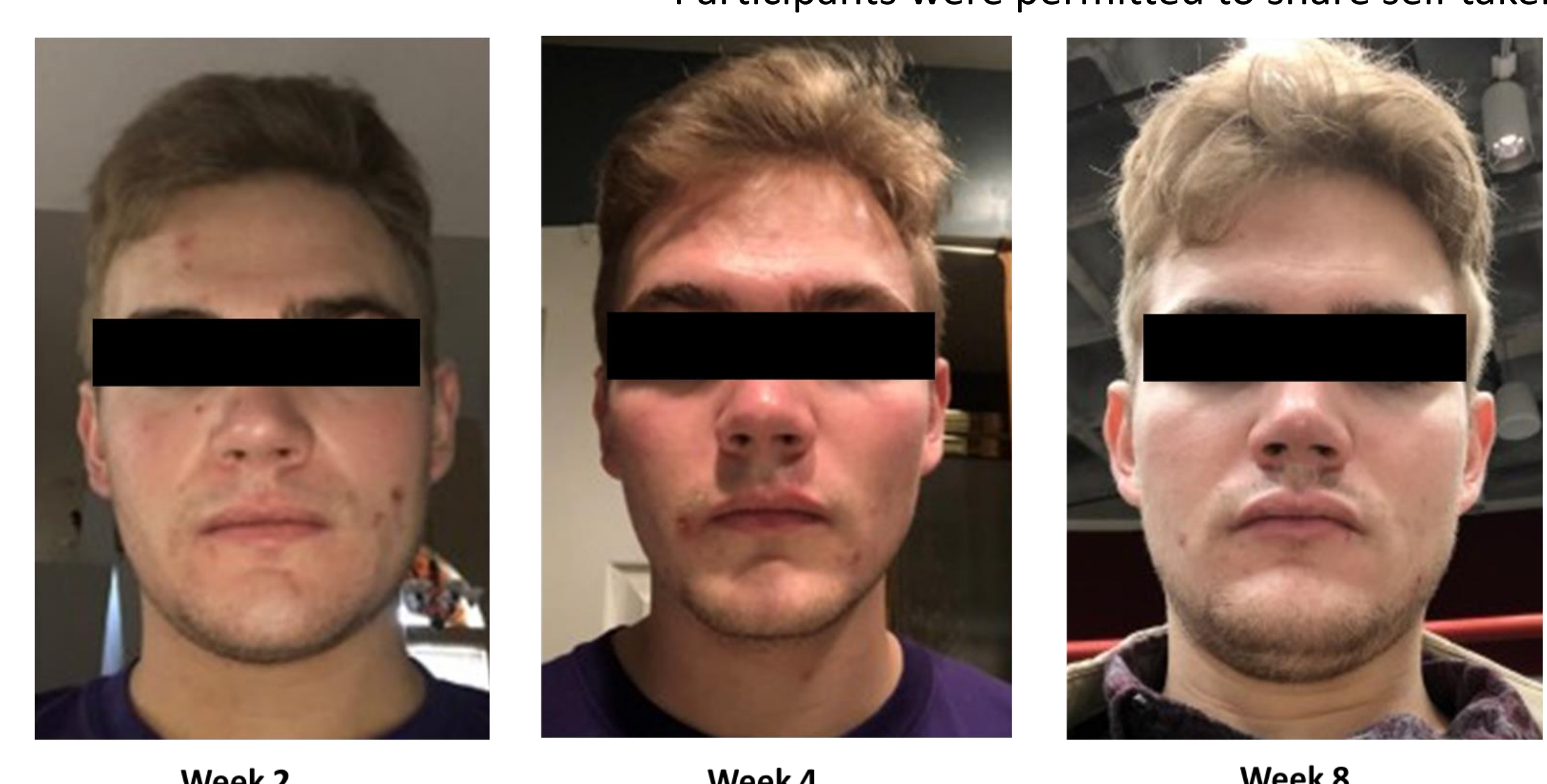

day to treat AV on his face, chest, and back

He was using a moisturizer, but provided no details as to the type or order of application (lo

Participants were asked to rate a number of qualities of tazarotene $0.1 \%$ largely as excellent or moisturizing. However, given that topical retinoids have been historically "moisturizing" as excellent or good speaks favorably to the novel tazarotene $0.1 \%$ foam vehicle.
Patients were asked to rate their satisfaction with tazarotene considered to be drying, a total of $32 \%$ of respondents ranking
Treatment Formulation Rating $\left(\mathrm{n}=371^{*}\right)$ $56 \%$

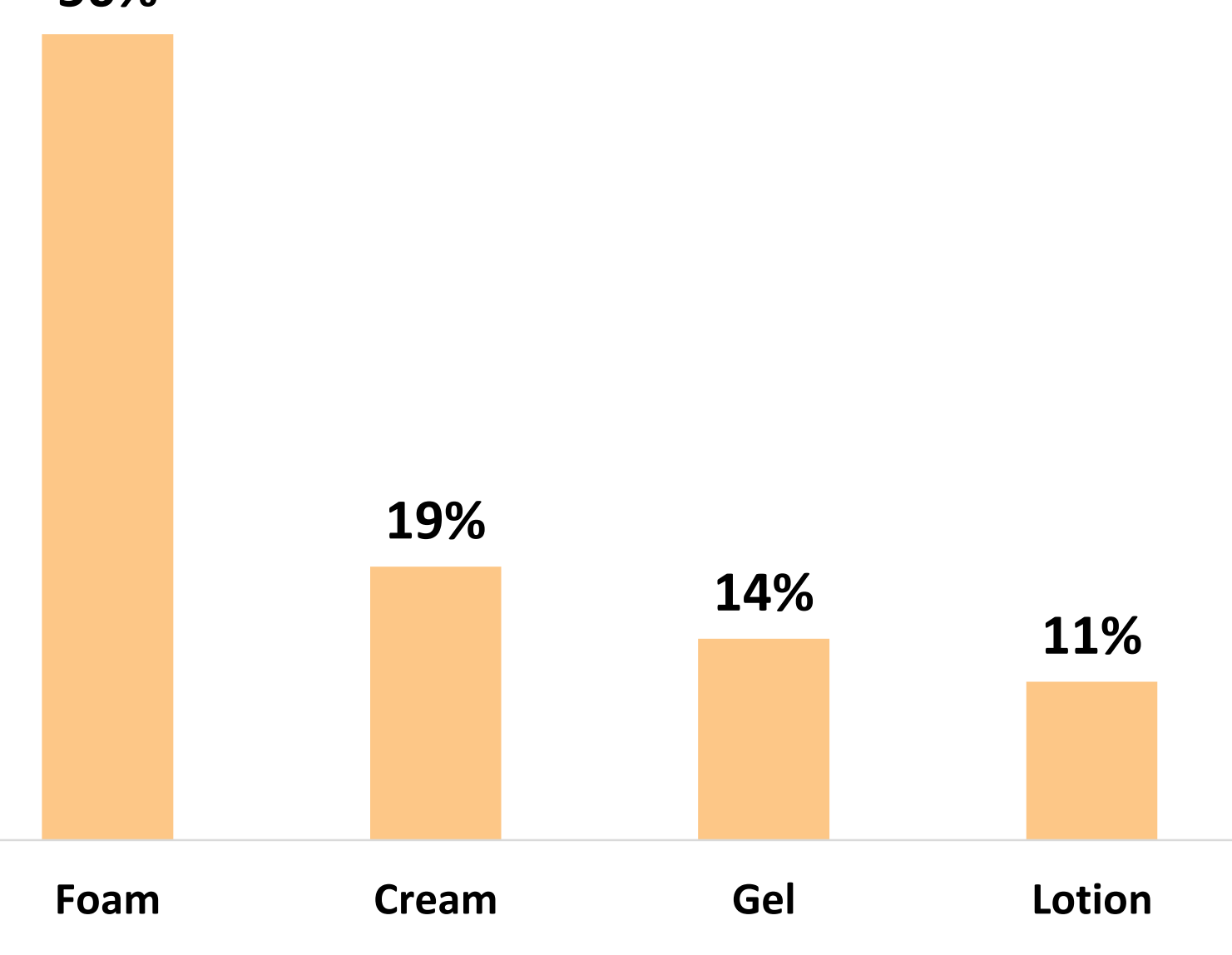

Participants were asked to rank topical vehicles on a scale of 1 to 5 , with 1 being the most preferred formulation and 5 received the highest ranking by far, with $56 \%$ of respondents rating it as "most preferred". This is between 2-5 times greater than the other vehicles, with the next highest bein
cream at only $19 \%$ of subjects rating it "most preferred".

\section{DISCLOSURE}

